SLAC-PUB-12103

September, 2006

\title{
Effect of the coupled-bunch modes on the longitudinal feedback system. ${ }^{*}$
}

\author{
S. Heifets, D. Teytelman \\ Stanford Linear Accelerator Center, Stanford University, Stanford, CA 94309, USA
}

\begin{abstract}
The Pedersen analysis [1, 2] of the low-level rf feedback system assumes that all bunches oscillate in phase what corresponds to the lowest coupled bunch mode. This analysis is extended here to take into account all other coupled-bunch modes what might be important for the strongly detuned cavities in large storage rings such as PEP-II.
\end{abstract}

\section{Introduction}

The Pedersen analysis [1, 2] of the low-level rf feedback system gives a solid ground for the design of the low-level rf feedback (FB) system and has been used for analysis and modeling of the FB in PEP-II B-factory. The analysis assumes that all bunches oscillate in phase what corresponds to the lowest coupled bunch mode. This analysis is extended here to take into account all other coupled-bunch modes what might be important for the strongly detuned cavities in large storage rings such as PEP-II.

For completeness and to define notations we, first, reproduce the basic definitions of the steady-state parameters of the rf FB system. In the derivation of the beam response, all coupled-bunch (CB) modes are taken into account and their effect is included in the analysis of the FB stability.

\section{Cavity voltage}

A rf cavity can be described as an LC contour excited by the power from a klystron and the beam current. Following P. Wilson [3] and G. Kraft [4], we describe the time dependence of the cavity voltage $V_{c}(t)$ by the equation 


$$
\left(\frac{d^{2}}{d t^{2}}+\frac{\omega_{c}}{Q_{L}} \frac{d}{d t}+\omega_{c}^{2}\right) V_{c}(t)=\frac{\omega_{c} R_{L}}{Q_{L}} \frac{d I_{t o t}}{d t} .
$$

Here $\omega_{c} / 2 \pi$ is the fundamental frequency of the cavity, $Q_{0}$ and $R_{0}$ are the quality factor and shunt impedance of the cavity, and $Q_{L}$ and $R_{L}$ are the loaded $Q$-factor and loaded shunt impedance, depending on the rf coupling parameter $\beta$,

$$
Q_{L}=\frac{Q_{0}}{1+\beta}, \quad R_{L}=\frac{R_{0}}{1+\beta} .
$$

The cavity is excited by the total current $I_{t o t}=I_{g}(t)-I_{B}(t)$, where $I_{g}$ and $I_{B}$ are the excitation current of the generator and the beam current, respectively.

Consider the rf cavity with the rf feedback system (FB). The FB loop is shown in Fig. (1). The voltage on the cavity is generated by the generator current $I_{g}$ and the beam current $I_{B}$ and is defined by the cavity impedance $Z_{c}$ as it is followed from Eq. (1):

$$
\begin{aligned}
& V_{c}(\omega)=Z_{C}(\omega)\left(I_{g}(\omega)-I_{B}(\omega)\right), \\
& Z_{c}(\omega)=\frac{R_{L}}{1-i Q_{L}\left(\frac{\omega}{\omega_{c}}-\frac{\omega_{c}}{\omega}\right)} .
\end{aligned}
$$

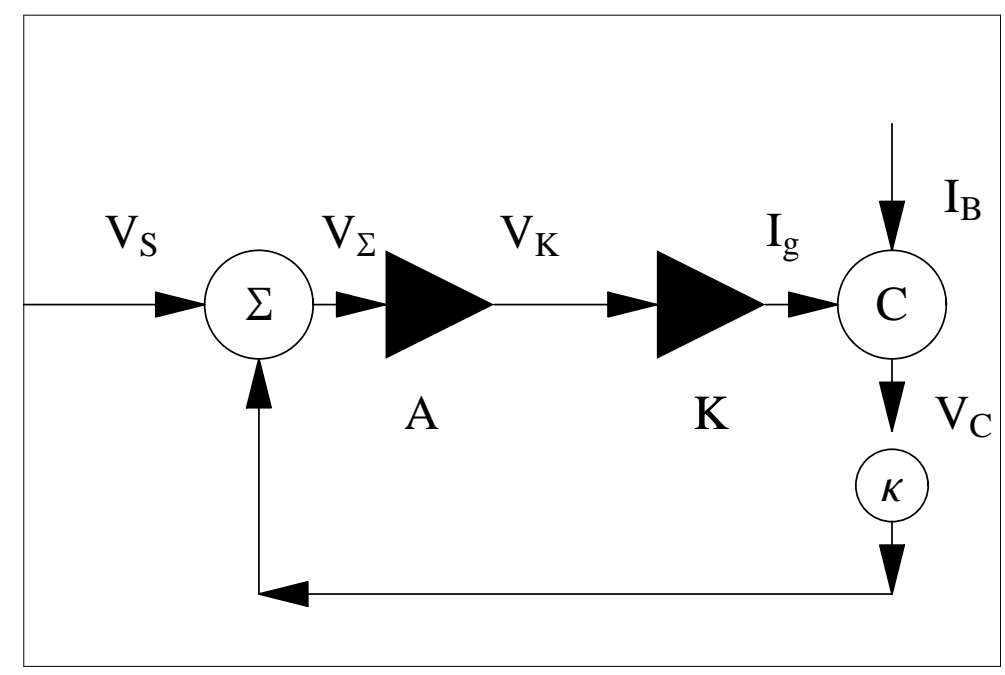

Figure 1: Schematic of the longitudinal rf FB system.

We use the time dependence of all quantities at the frequency $\omega$ in the form $e^{-i \omega t}$.

The input voltage on the amplifier is $V_{\Sigma}=V_{s}-\kappa V_{c}$, where $V_{s}$ is the setup voltage and $\kappa$ is a real parameter of the attenuator, $\kappa \simeq 10^{-6}$. After the amplifier with the transfer 
function $T_{A}$, the input voltage to the klystron is $V_{K}=T_{A} V_{\Sigma}$. The output generator current feeding the rf cavity is

$$
I_{g}=\frac{V_{K}}{T_{K}}=\frac{T_{A}}{T_{K}}\left(V_{s}-\kappa V_{c}\right) .
$$

Substituting Eq. (4) into Eq. (3) we obtain

$$
\begin{aligned}
& V_{c}(\omega)=\frac{(G / \kappa) V_{s}(\omega)-R_{L} I_{B}(\omega)}{1-2 i Q_{L}\left(\frac{\omega-\omega_{c}}{\omega_{c}}\right)+G}, \\
& I_{g}(\omega)=\frac{G}{\kappa R_{L}} V_{s}(\omega)-G \frac{V_{c}(\omega)}{R_{L}} .
\end{aligned}
$$

Here we use notation $G$ for the FB transfer function defined in terms of the electronic gain $H$ and the delay time $\tau_{d}$ as

$$
G=\kappa \frac{T_{A}}{T_{K}} R_{L}=H e^{i\left(\omega-\omega_{c}\right) \tau_{d}} .
$$

Parameters $H$ and $\tau_{d}$ are, approximately, independent of frequency. For PEP-II, $H \simeq 6$ and $\tau_{d}=450 \mathrm{~ns}$.

The FB stability requires that the poles of $V_{c}(\omega)$ as function of complex $\omega$ have to be in the lower half plane. In other words, for the FB stability, the imaginary part of zeros of the denominator in Eq. (5) for $V_{c}(\omega)$ has to be negative. Result of the calculation for the PEP-II coupling $\beta_{R F}=3.6, Q_{L}=6956.5$, and delay time $\tau_{d}=450 \mathrm{~ns}$ is shown in Fig. (2). The FB system is stable for $H<\simeq 15$. The actual limit is lower and is defined by distortion of the cavity impedance at large $H$ [5], see Fig. (3).

\subsection{Steady-state conditions}

Consider the rf frequency $\omega_{g}=h \omega_{0}$, where $\omega_{0} /(2 \pi)$ is the revolution frequency, and $h$ is the harmonic number. We define quantities

$$
\begin{aligned}
& \mu=\left(\omega_{g}-\omega_{c}\right) \tau_{d}, \\
& \zeta=2 Q_{L} \frac{\omega_{g}-\omega_{c}}{\omega_{c}}, \\
& R_{H}=\frac{R_{L}}{1+H \cos \mu}, \\
& \tan \psi=\frac{\zeta-H \sin \mu}{1+H \cos \mu} .
\end{aligned}
$$

Let us define the amplitudes and phases ${ }^{\dagger}$

\footnotetext{
${ }^{\dagger} \mathrm{F}$. Pedersen uses phases $\phi_{L}, \phi_{B}$, and $\phi_{z}$ related to our $\phi_{c}, \phi_{s}$, and $\psi: \phi_{L}=-\phi_{c}, \pi / 2-\phi_{B}=\phi_{s}$, $\phi_{z}=\psi$.
} 


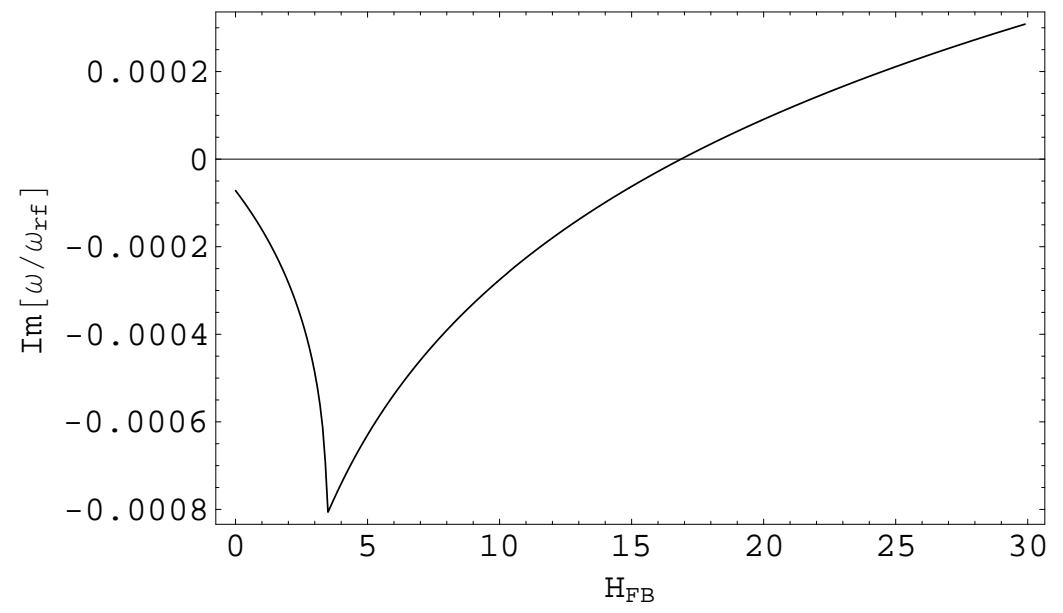

Figure 2: Imaginary part of the poles of $V_{c}(\omega)$ as function of the FB gain $H$. The FB is stable if imaginary part shown in the plot is negative.

$$
\begin{aligned}
& V_{c}(t)=V \cos \left(\omega_{g} t+\phi_{c}\right)=\frac{1}{2} \hat{V}_{c} e^{-i \omega_{g} t}+c . c ., \quad \hat{V}_{c}=V e^{-i \phi_{c}}, \\
& I_{B}(t)=2 I_{d c} \cos \left(\omega_{g} t+\phi_{c}-\phi_{s}\right)=\frac{1}{2} \hat{I}_{B} e^{-i \omega_{g} t}+c . c ., \quad, \quad \hat{I}_{B}=2 I_{d c} e^{-i\left(\phi_{c}-\phi_{s}\right)}, \\
& I_{g}(t)=\frac{1}{2} \hat{I}_{g} e^{-i \omega_{g} t}+c . c . \\
& V_{s}(t)=\frac{1}{2} \hat{V}_{s} e^{-i \omega_{g} t}+c . c .
\end{aligned}
$$

The common phase is arbitrary and we assume that $\hat{I}_{g}$ is real.

In this notations and at $\omega=\omega_{g}$, Eq. (5) takes the form

$$
\begin{aligned}
& \hat{V}_{c}=Z_{H}\left(\omega_{g}\right) \hat{I}_{t o t}, \\
& \hat{I}_{t o t}=\frac{H}{\kappa R_{L}} \hat{V}_{s} e^{i \mu}-\hat{I}_{B} .
\end{aligned}
$$

The impedance $Z_{H}\left(\omega_{g}\right)$ at the rf frequency is

$$
Z_{H}\left(\omega_{g}\right)=R_{H} \cos (\psi) e^{i \psi}
$$

Hence, the detuning angle $\psi$ is the angle between $V_{c}$ and the total current $I_{\text {tot }}$.

A bunch crosses the cavity at the moments when $I_{B}(t)$ is maximum and sees the accelerating voltage $V_{a c c}=V \cos \left(\phi_{s}\right)$. The synchronous phase $\phi_{s}$, therefore, is defined 


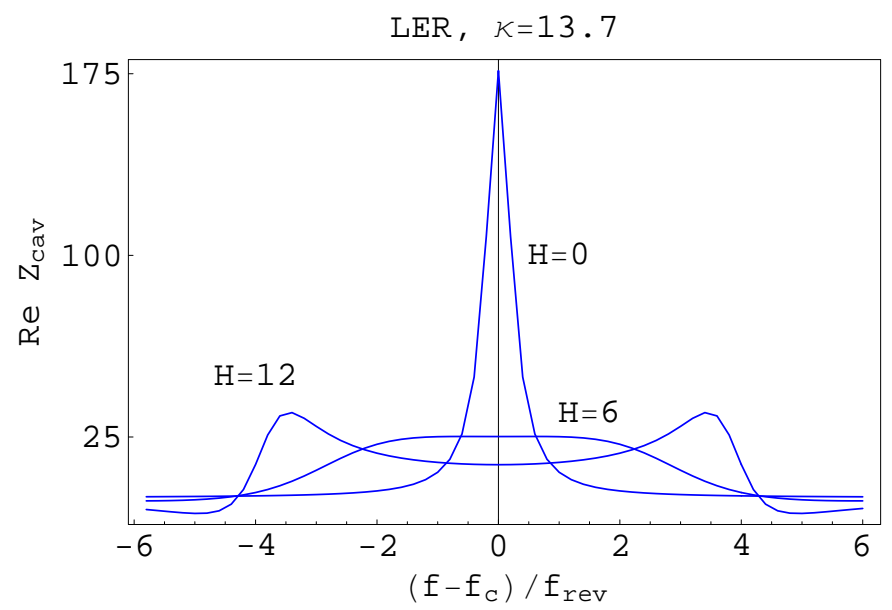

Figure 3: Real part of the impedance for several values of the FB gain $H$. The impedance starts to grow at large $H$ and can cause instability at the rf sidebands for detuned cavities.

by the energy loss per particle per turn due to synchrotron radiation $U_{S R}$ and the HOM losses $U_{H O M}=N_{b} e^{2} \kappa_{l}$ proportional to the bunch population $N_{B}$ and the total loss factor $\kappa_{l}$ (except of the loss factor of the fundamental mode).

For the $n_{\text {cav }}$ cavities in the ring,

$$
n_{\text {cav }} V \cos \phi_{s}=U_{S R}+U_{H O M} .
$$

For the beam stability, the accelerating voltage has to have a negative slope, $d V_{a c c} / d \phi_{s}<$ 0 . In this case, a particle having larger energy and shifted above the transition energy to the tail of the bunch sees the accelerating voltage smaller than the equilibrium particle. The derivative $d V_{a c c} / d \phi_{s}$ is taken for fixed parameters $I_{d c}, \omega_{g}, \omega_{c}, \kappa, \beta, H, \tau_{d}$ and the setup voltage $V_{s}$. Therefore, $R_{L}, Q_{L}, R_{H}, \mu$ and $\psi$ are also constant, while $\left|\hat{V}_{c}\right|$ and $\phi_{c}$ vary. Taking derivative of $V_{c}$ given by the first of Eqs. (9), we get

$$
\frac{d\left|\hat{V}_{c}\right|}{d \phi_{s}}=i\left|\hat{V}_{c}\right| \frac{d \phi_{c}}{d \phi_{s}}+2 i I_{d c} R_{H} \cos \psi e^{i\left(\phi_{s}+\psi\right)}\left(\frac{d \phi_{c}}{d \phi_{s}}-1\right), \quad\left|\hat{V}_{c}\right|=V .
$$

The real and imaginary parts give two equations

$$
\begin{aligned}
\frac{d \phi_{c}}{d \phi_{s}} & =\frac{Y_{H} \cos \psi \cos \left(\psi+\phi_{s}\right)}{1+Y_{H} \cos \psi \cos \left(\psi+\phi_{s}\right)}, \\
\frac{d\left|\hat{V}_{c}\right|}{d \phi_{s}} & =\left|\hat{V}_{c}\right| \tan \left(\psi+\phi_{s}\right) \frac{d \phi_{c}}{d \phi_{s}} .
\end{aligned}
$$

Here 


$$
Y_{H}=\frac{Y_{L}}{1+H \cos \mu}, \quad Y_{L}=\frac{2 I_{d c} R_{L}}{V} .
$$

The Robinson condition of stability takes the form

$$
\sin \left(\phi_{s}\right)>\frac{Y_{H}}{2} \sin (2 \psi)
$$

Without $\mathrm{FB}, H=0$, it takes the standard form. Limitation on the beam current are shown in Fig. (4). At a fixed voltage, the maximum current increases with the FB on. However, as it was mentioned above, the maximum gain $H$ is limited by the stability of the rf sidebands, see Fig. (3).

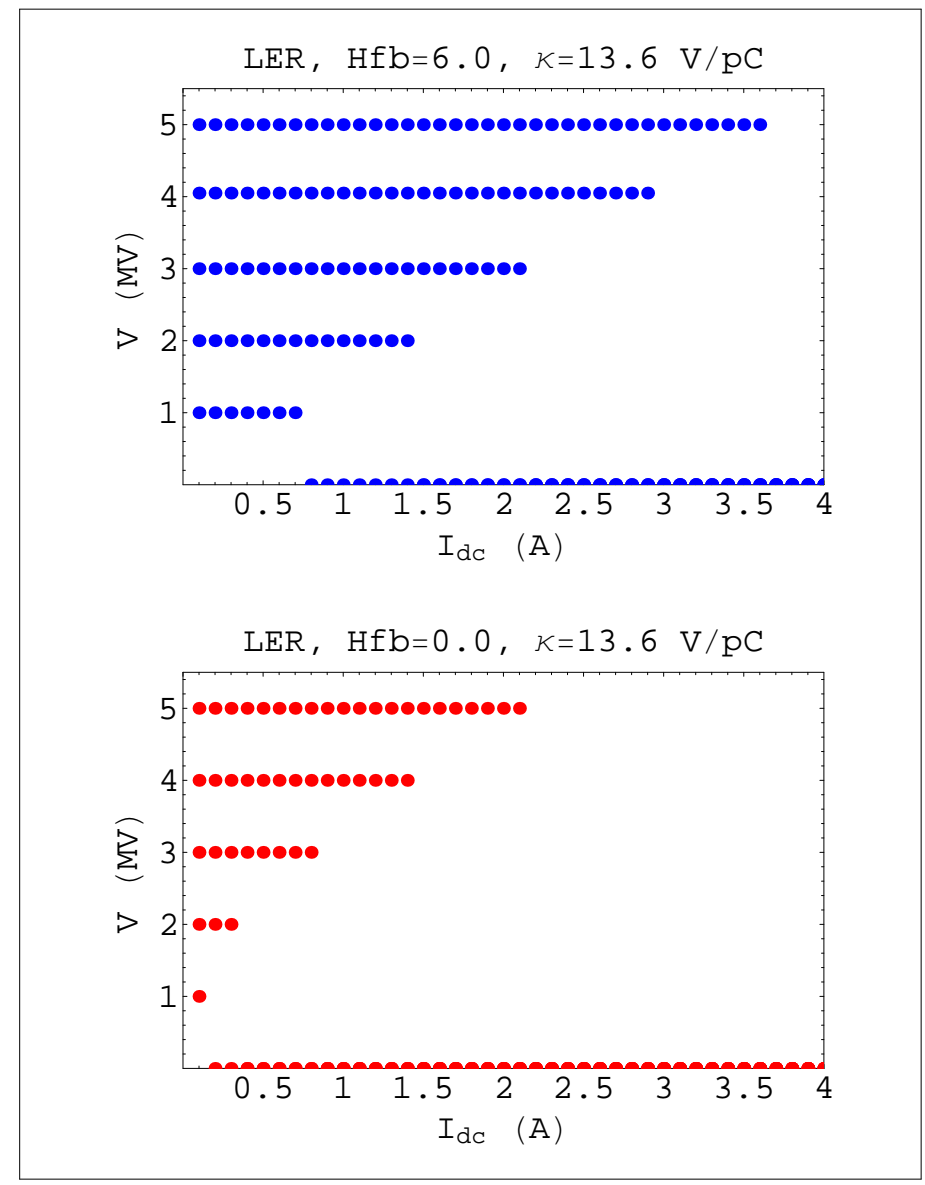

Figure 4: For each rf voltage of the PEP-II LER, the beam currents corresponding to Robinson criterion of stability are shown by dots. Upper plot: with the FB $H_{g}=6.0$, bottom plot with the FB turned off, $H_{g}=0$. 


\subsection{Optimum conditions}

The optimum conditions correspond to the minimum of reflected power

$$
P_{r}=\frac{\beta}{2(1+\beta) R_{L}}\left|\hat{V}_{c}-\frac{R_{L}(1+\beta)}{2 \beta} I_{g}\right|^{2}
$$

Substituting

$$
\hat{I}_{g}=\hat{V}_{c} / Z_{c}+\hat{I}_{B}
$$

from Eq. (3), $\hat{V}_{c}=\left|\hat{V}_{c}\right| e^{-i \phi_{c}}, \hat{I}_{B}=2 I_{d c} e^{i\left(\phi_{s}-\phi_{c}\right)}$, and using definition of $Y_{L}$, we get

$$
P_{r}=\frac{(1+\beta)\left|\hat{V}_{c}\right|^{2}}{8 \beta R_{L}}\left|\frac{\beta-1}{\beta+1}-Y_{L} \cos \phi_{s}+i\left(\zeta-Y_{L} \sin \phi_{s}\right)\right|^{2} .
$$

$P_{r}$ is minimum if the imaginary part is zero. This defines the optimum detuning

$$
\zeta=2 Q_{L} \frac{\omega_{g}-\omega_{c}}{\omega_{c}}=Y_{L} \sin \phi_{s}
$$

and the detuning angle $\psi$ by Eq. (7).

The reflected power in the optimum

$$
P_{r}=\frac{(1+\beta)\left|\hat{V}_{c}\right|^{2}}{8 \beta R_{L}}\left|\frac{\beta-1}{\beta+1}-Y_{L} \cos \phi_{s}\right|^{2} .
$$

is zero for fixed cavity voltage only at certain current, when

$$
Y_{L} \cos \phi_{s}=\frac{\beta-1}{\beta+1}
$$

Eqs. (19),(17) give

$$
\hat{I}_{g}=\frac{\left|\hat{V}_{c}\right|}{R_{L}} e^{-i \phi_{c}}\left(1+Y_{L} \cos \phi_{s}\right) .
$$

By definition, $\hat{I}_{g}$ is real. Hence, $\phi_{c}$ in the optimum is zero.

Usually, the FB system operates to produce a given cavity voltage $V_{c}$ for a beam current $I_{B}$. Then Eq. (5) taken at $\omega=\omega_{g}$ defines the reference voltage $V_{s}$,

$$
V_{s}\left(\omega_{g}\right)=\frac{\kappa V_{c}}{H} e^{-i \mu}\left\{1-i \zeta+H e^{i \mu}+Y_{L} e^{i \phi_{s}}\right\}
$$

where $\mu$ and $\zeta$ are defined by Eq. (7). 


\section{Analysis of small perturbations}

The steady-state cavity voltage $V^{(0)}(t)=\frac{1}{2} \hat{V}^{0} e^{-i \omega_{g} t}+$ c.c. is the sum of voltages induced by the setup voltage $V_{c a v}^{(s)}$, the voltage $V_{c a v}^{(B)}$ induced by the beam current $I_{B}$, and of the $\mathrm{FB} V_{c a v}^{(F B)}$,

$$
V^{0}=V_{c a v}^{(s)}-V_{c a v}^{(B)}-V_{c a v}^{(F B)} .
$$

If there is a beam perturbation $\delta I_{B}$, the setup voltage $V_{c a v}^{(s)}$ do not change, but the cavity voltage and the total current change.

Following F. Pedersen [1], we write the small variations of the voltage and the total current around the steady-state as

$$
\begin{array}{ll}
\delta V_{c}(t)=\frac{1}{2} \hat{V}_{c} e^{-i \omega_{g} t} U(t)+c . c ., & U(t)=u[t]-i \Phi[t], \\
\delta I_{t o t}(t)=\frac{1}{2} \hat{I}_{t o t} e^{-i \omega_{g} t} J[t]+c . c ., & J(t)=a(t)-i b(t) .
\end{array}
$$

where the slow functions of time $U(t)$ and $J(t)$ are split in the real and imaginary parts.

$\hat{V}_{c}$ and $\hat{I}_{t o t}$ are related by Eq. (9), $\hat{V}_{c}=Z_{H}\left(\omega_{g}\right) \hat{I}_{t o t}$. Eq. (25) gives for Fourier harmonics

$$
\begin{aligned}
& \delta V_{c}(\omega)=\frac{1}{2} \hat{V}_{c} U_{-}\left(\omega-\omega_{g}\right)+\frac{1}{2} \hat{V}_{c}^{*} U_{+}\left(\omega+\omega_{g}\right), \quad U_{ \pm}(\omega)=u(\omega) \pm i \Phi(\omega), \\
& \delta I_{\text {tot }}(\omega)=\frac{1}{2} \hat{I}_{\text {tot }} J_{-}\left(\omega-\omega_{g}\right)+\frac{1}{2} \hat{I}_{\text {tot }}^{*} J_{+}\left(\omega+\omega_{g}\right), \quad J_{ \pm}(\omega)=a(\omega) \pm i b(\omega),
\end{aligned}
$$

where $U(\omega)=u(\omega)-i \Phi(\omega), J(\omega)=a(\omega)-i b(\omega), u(-\omega)=u^{*}(\omega), \Phi(-\omega)=\Phi^{*}(\omega)$, $a(-\omega)=a^{*}(\omega), b(-\omega)=b^{*}(\omega)$ and the star indicates complex conjugation.

The perturbation $\delta I_{\text {tot }}(\omega)=\delta I_{g}-\delta I_{B}$ causes $\delta V_{c}(\omega)=Z_{c}(\omega) \delta I_{\text {tot }}(\omega)$. Because, the reference voltage $V_{s}$ is fixed, then $\delta V_{\Sigma}=-\kappa Z_{c} \delta I_{\text {tot }}$, see Fig.(1). The variation $\delta V_{\Sigma}$ propagates giving $\delta V_{K}=T_{A} \delta V_{\Sigma}$ and $\delta I_{g}=\delta V_{K} / T_{K}$. Hence,

$$
\left(1+\kappa \frac{T_{A}}{T_{K}} Z_{c}(\omega)\right) \delta I_{\text {tot }}(\omega)=-\delta I_{B}(\omega)
$$

or

$$
\begin{aligned}
& \delta I_{t o t}(\omega)=-\frac{Z_{H}(\omega)}{Z_{c}(\omega)} \delta I_{B}(\omega), \\
& \delta V_{c}(\omega)=-Z_{H}(\omega) \delta I_{B}(\omega)=Z_{c}(\omega) \delta I_{\text {tot }}(\omega) .
\end{aligned}
$$


Combining Eq. (26) and the second Eq. (28) produces the first set of F. Pedersen's relations

$$
\begin{aligned}
& u(\omega)=G_{+}(\omega) a(\omega)-i G_{-}(\omega) b(\omega), \\
& \Phi(\omega)=i G_{-}(\omega) a(\omega)+G_{+}(\omega) b(\omega),
\end{aligned}
$$

where

$$
G_{ \pm}=\frac{1}{2}\left[ \pm \frac{Z_{c}\left(\omega-\omega_{g}\right)}{Z_{H}^{*}\left(\omega_{g}\right)}+\frac{Z_{c}\left(\omega+\omega_{g}\right)}{Z_{H}\left(\omega_{g}\right)}\right] .
$$

Note that $G_{ \pm}^{*}(-\omega)= \pm G_{ \pm}(\omega)$.

The beam dynamics relates $\delta V_{c}$ to $\delta I_{B}(\omega)$ and, through the first Eq. (28), to $\delta I_{t o t}$. This is considered in the next section.

\subsection{Beam dynamics}

Let us consider a train of $n_{b}$ ultra-relativistic bunches in the ring with the circumference $2 \pi R=c T_{0}=2 \pi c / \omega_{0}$. For the frequencies within the bandwidth of the FB system, bunches can be considered as point-like macro-particles. In an equilibrium, the center of the $n$-th bunch is at the distance $s_{n}=(n-1) s_{b}$ from the head of the train. The position of the bunch in the ring $s_{n}$ is

$$
s_{n}(t)=c\left(t-t_{0}\right)-(n-1) s_{b}+\zeta_{n}(t)
$$

where $c$ is velocity of light, $t_{0}=$ const, and $\zeta_{n}>0$ means the displacement of the bunch center to the head of the train. $\zeta_{n}(t)$ describes the synchrotron oscillations. The amplitude of oscillations may be itself a slow function of time. The constant longitudinal offset of the bunches in the train is included in $t_{0}$.

On the $k$-th turn, the $n$-th bunch centroid arrives to an rf cavity located at $s=0$ in the ring at the moment $t_{k, n}=k T_{0}+t_{0}+(n-1) s_{b} / c$.

The beam current exciting a cavity located at $s=0$ around the ring is

$$
\begin{aligned}
& I_{B}(t)=\frac{I_{d c} c T_{0}}{n_{b}} \sum_{n=1}^{n_{b}} \sum_{k=-\infty}^{\infty} \delta\left[c\left(t-t_{0}\right)-(n-1) s_{b}+\zeta_{n}(t)+k c T_{0}\right]= \\
& \frac{I_{d c} T_{0}}{n_{b}} \sum_{n=1}^{n_{b}} \int \frac{d \omega}{2 \pi} \sum_{k=-\infty}^{\infty} \exp \left[-i \omega\left(t-t_{0}-(n-1) \frac{s_{b}}{c}+\frac{\zeta_{n}(t)}{c}+k T_{0}\right)\right] .
\end{aligned}
$$

Using the identity

$$
\sum_{k=-\infty}^{\infty} \exp \left[-i k \omega T_{0}\right]=\frac{2 \pi}{T_{0}} \sum_{k=-\infty}^{\infty} \delta\left[\omega-k \omega_{0}\right],
$$


Eq. (32) in the linear over $\zeta$ approximation can be written in the form

$$
I_{B}(t)=\frac{I_{d c}}{n_{b}} \sum_{n=1}^{n_{b}} \sum_{k=-\infty}^{\infty} \exp \left[i k \omega_{0}\left(t-t_{0}-(n-1) \frac{s_{b}}{c}\right)\right]\left(1+i \frac{k \omega_{0}}{c} \zeta_{n}(t)\right) .
$$

We assume the uniform fill of bunches, $\omega_{0} s_{b} / c=2 \pi / n_{b}$, expanding $\zeta_{n}(t)$ over the coupled-bunch eigen modes $X_{n}^{\mu}$ with amplitudes $A_{\mu}(t), A_{\mu}^{*}(t)=A_{-\mu}(t), \mu=0,1, . ., n_{b}-1$,

$$
\zeta_{n}(t)=\sum_{\mu=0}^{n_{b}-1} A_{\mu}(t) X_{n}^{\mu}, \quad X_{n}^{\mu}=\frac{1}{\sqrt{n_{b}}} e^{2 \pi i(n-1) \mu / n_{b}} .
$$

Now we can use the identity

$$
\sum_{n=1}^{n_{b}} \exp \left[-i k \omega_{0}(n-1) s_{b} / c\right]=n_{b} \sum_{p=-\infty}^{\infty} \delta_{k, p n_{b}}
$$

and

$$
\sum_{n=1}^{n_{b}}\left(X_{n}^{\mu}\right) e^{-i k \omega_{0}(n-1) s_{b} / c}=\sqrt{n_{b}} \sum_{p=-\infty}^{\infty} \delta_{k-\mu, p n_{b}},
$$

where $\delta_{i, k}=1$ for $i=k$ and $\delta_{i, k}=0$ otherwise.

Eq. (32) is transformed to

$$
I_{B}(t)=I_{d c} \sum_{p=-\infty}^{\infty} e^{i p n_{b} \omega_{0}\left(t-t_{0}\right)}\left\{1+i \frac{\omega_{0}}{c \sqrt{n_{b}}} \sum_{\mu}\left(p n_{b}+\mu\right) A_{\mu}(t) e^{i \mu \omega_{0}\left(t-t_{0}\right)}\right\} .
$$

Here harmonics $p= \pm h / n_{b}$ corresponds to the steady-state beam current. Hence,

$$
\omega_{g} t_{0}=\left(\phi_{s}-\phi_{c}\right)
$$

All other harmonics are separated in frequency by $n_{b} \omega_{0}$ and can be ignored for the analysis of the low-level FB with the bandwidth limited to few revolution harmonics.

The perturbation of the beam current is given by the second term in Eq. (38),

$$
\delta I_{B}(t)=i \frac{I_{d c} \omega_{g}}{c \sqrt{n_{b}}} \sum_{\mu} \sum_{p}\left(\frac{p n_{B}+\mu}{h}\right) e^{i\left(\frac{p n_{b}+\mu}{h}\right)\left(\omega_{g} t-\phi_{s}\right)} A_{\mu}(t) .
$$

The Fourier harmonics for the optimum $\phi_{c}=0$ are

$$
\delta I_{B}(\omega)=i \frac{I_{d c} \omega_{g}}{c \sqrt{n_{b}}} \sum_{\mu} \sum_{p}\left(\frac{p n_{B}+\mu}{h}\right) e^{-i\left(\frac{p n_{b}+\mu}{h}\right) \phi_{s}} A_{\mu}\left[\omega+\left(p n_{b}+\mu\right) \omega_{0}\right]
$$




\subsection{Synchrotron motion}

The synchrotron oscillations are defined by the rf voltage and the longitudinal wake $W(t)$. The later produces two effects: it changes the synchrotron frequency and generates bunch coupling.

Consider equation of motion for the $n$-th bunch in the linear approximation over $\zeta_{n}$ taking into account the wake and the perturbation of the rf voltage $\delta V_{c}(t)$,

$$
\begin{aligned}
& \frac{d^{2} \zeta_{n}(t)}{d t^{2}}+\omega_{s, 0}^{2} \zeta_{n}(t)=\sum_{k=-\infty}^{\infty} \delta\left[t-t_{k, n}\right]\left\{-\left(\frac{\alpha c e n_{\text {cav }}}{E}\right) \delta V_{c}(t)+\right. \\
& \left.\left(\frac{\alpha N_{b} e^{2}}{E}\right) \sum_{k^{\prime}=-\infty}^{\infty} \sum_{m=1}^{n_{b}} W^{\prime}\left[t_{k, n}-t_{k^{\prime}, m}\right]\left(\zeta_{m}\left(t_{k^{\prime}, m}\right)-\zeta_{n}\left(t_{k, n}\right)\right)\right\} .
\end{aligned}
$$

Here $N_{B}$ and $E$ are the bunch population and the beam energy, respectively, $\alpha$ is the momentum compaction factor, and $\omega_{s}^{0} / 2 \pi$ is the zero-current synchrotron frequency. The longitudinal wake per turn is defined to be zero for $t<0$, and the prime in $W^{\prime}[t]$ means derivative over $t$. The longitudinal impedance $Z(\omega)$ is the Fourier component of the wake $W[t]$ analytic in the upper half plane of $\omega$.

It is well known [7], that for equidistant bunches and $\delta V_{c}=0$, expansion Eq. (35) in the orthogonal modes $X_{n}^{\mu}$ reduces Eq. (42) to independent equations for the constant in time amplitudes $A_{\mu}$. The same is true for a slow perturbation $\delta V_{c}(t) \neq 0$ giving equations for the amplitudes $A_{\mu}(t)$ slowly varying in time.

The Fourier harmonics $A_{\mu}(\omega)$,

$$
A_{\mu}(t)=\int d \omega /(2 \pi) A_{\mu}(\omega) e^{-i \omega t}
$$

satisfy equations

$$
\left(\omega_{s, 0}^{2}-\omega^{2}\right) A_{\mu}(\omega)=G_{\mu}(\omega),
$$

where

$$
\begin{aligned}
& G_{\mu}(\omega)=\int d t e^{i \omega t} \sum_{k=-\infty}^{\infty} \sum_{n=1}^{n_{b}}\left[X_{n}^{\mu}\right]^{*} \delta\left[t-t_{k n}\right]\left\{-\left(\frac{\alpha c e n_{\text {cav }}}{E}\right) \delta V_{c}(t)+\right. \\
& \left.\left(\frac{\alpha N_{b} e^{2}}{E}\right) \sum_{k^{\prime}=-\infty}^{\infty} \sum_{m=1}^{n_{b}} W^{\prime}\left[t_{k, n}-t_{k^{\prime}, m}\right]\left(\zeta_{m}\left(t_{k^{\prime}, m}\right)-\zeta_{n}\left(t_{k, n}\right)\right)\right\} .
\end{aligned}
$$

Substituting Fourier expansion for $\delta V(t)$, for the wake, and $\zeta(T)$, we can carry out integration over $t$. That gives 


$$
\begin{aligned}
& G_{\mu}(\omega)=-\left(\frac{\alpha c e n_{c a v} \omega_{0}}{2 \pi E}\right) \sqrt{n_{b}} \sum_{p=-\infty}^{\infty} \delta V_{c}\left[\omega+\left(p n_{b}-\mu\right) \omega_{0}\right] e^{-i\left(p n_{b}-\mu\right) \omega_{0} t_{0}} \\
& -i\left(\frac{\alpha N_{b} n_{b} e^{2}}{E}\right)\left(\frac{\omega_{0}}{2 \pi}\right)^{2} \sum_{p, p^{\prime}=-\infty}^{\infty} \sum_{\mu^{\prime}=0}^{n_{b}-1} A_{\mu^{\prime}}\left[\omega-\left(p+p^{\prime}\right) n_{b} \omega_{0}-\left(\mu-\mu^{\prime}\right) \omega_{0}\right] e^{i\left[\left(p+p^{\prime}\right) n_{b}-\mu^{\prime}+\mu\right] \omega_{0} t_{0}} \\
& {\left[\left(\omega-p n_{b} \omega_{0}-\mu \omega_{0}\right) Z\left(\omega-\left(p n_{b}+\mu\right) \omega_{0}\right)-p^{\prime} n_{b} \omega_{0} Z\left(p^{\prime} n_{b} \omega_{0}\right)\right] .}
\end{aligned}
$$

The amplitude $A_{\mu}(\omega) \neq 0$ for low frequencies, $|\omega|<<n_{b} \omega_{0}$. Therefore, we can drop all terms with $p^{\prime} \neq p$. The impedance dependent diagonal term $\mu^{\prime}=\mu$ can be combined with the term $\omega_{s, 0}^{2}$ in the LHS of Eq. (44) redefining the synchrotron frequency of the mode $\mu$,

$$
\begin{aligned}
& \omega_{\mu, s}^{2}=\omega_{s, 0}^{2}+\delta \omega_{s, \mu}^{2} \\
& \delta \omega_{s, \mu}^{2}=i\left(\frac{\alpha N_{b} n_{b} e^{2}}{E}\right)\left(\frac{\omega_{0}}{2 \pi}\right)^{2} \\
& \sum_{p}^{\infty}\left[\left(\omega+p n_{b} \omega_{0}-\mu \omega_{0}\right) Z\left(\omega+\left(p n_{b}-\mu\right) \omega_{0}\right)-p n_{b} \omega_{0} Z\left(p n_{b} \omega_{0}\right)\right]
\end{aligned}
$$

Eq. (46) takes the form

$$
\begin{aligned}
& G_{\mu}(\omega)=-\left(\frac{\alpha c e n_{c a v} \omega_{0}}{2 \pi E}\right) \sqrt{n_{b}} \sum_{p=-\infty}^{\infty} \delta V_{c}\left[\omega+\left(p n_{b}-\mu\right) \omega_{0}\right] e^{-i\left(p n_{b}-\mu\right) \omega_{0} t_{0}} \\
& -\delta \omega_{s, \mu}^{2} \sum_{\mu^{\prime} \neq \mu} A_{\mu^{\prime}}\left[\omega-\left(\mu-\mu^{\prime}\right) \omega_{0}\right] e^{-i\left(\mu^{\prime}-\mu\right) \omega_{0} t_{0}} .
\end{aligned}
$$

The last sum (proportional to the synchrotron tune shift $\delta \omega_{s, \mu}^{2}$ ) is zero for amplitudes $A_{\mu}$ independent of time. For slow dependence on time it produces coupling of the amplitudes $A_{\mu}$. Usually, however, $\delta \omega_{s, \mu}$ is small, $\delta \omega_{s, \mu}<<\omega_{s, 0}$, and we can neglect coupling. In this case,

$$
G_{\mu}(\omega)=-\left(\frac{\alpha c e n_{c a v} \omega_{0}}{2 \pi E}\right) \sqrt{n_{b}} \sum_{p=-\infty}^{\infty} \delta V_{c}\left[\omega+\left(p n_{b}-\mu\right) \omega_{0}\right] e^{-i\left(p n_{b}-\mu\right) \omega_{0} t_{0}} .
$$

Eq. (44) defines $A_{\mu}(\omega)$ in terms of Fourier harmonics of the perturbation $\delta V(\omega)$,

$$
A_{\mu}(\omega)=-\left(\frac{\alpha c e n_{c a v}}{E}\right) \frac{\omega_{0} \sqrt{n_{b}}}{2 \pi\left[\omega_{s \mu}^{2}-\omega^{2}\right]} \sum_{p=-\infty}^{\infty} \delta V_{c}\left[\left(p n_{b}-\mu\right) \omega_{0}+\omega\right] e^{-i\left(p n_{b}-\mu\right) \omega_{0} t_{0}} .
$$

The coefficient can be written in terms of $\omega_{s, 0}$,

$$
\left(\frac{\alpha c e n_{c a v}}{E}\right)=\left(\frac{\omega_{s, 0}^{2} c T_{0}}{\omega_{g} V_{c} \sin \left(\phi_{s}\right)}\right)
$$




\subsection{Beam transfer function}

In the optimum, $\phi_{c}=0$ and $\hat{V}_{c}=\hat{V}_{c}^{*}$. Substituting Eq. (50) in Eq. (41) we get for $\delta I_{B}(\omega)$ expression

$$
\delta I_{B}(\omega)=-i \frac{I_{d c}}{V_{c} \sin \left(\phi_{s}\right)} \sum_{p=-\infty}^{\infty} \sum_{p^{\prime}=-\infty}^{\infty} \sigma[\omega, p] \delta V_{c}\left[\omega+\left(p+p^{\prime}\right) n_{b} \omega_{0}\right] e^{-i\left(\frac{\left(p+p^{\prime}\right) n_{b}}{h}\right) \phi_{s}}
$$

where

$$
\sigma[\omega, p]=\sum_{\mu}\left(\frac{p n_{b}+\mu}{h}\right) \frac{\omega_{s 0}^{2}}{\omega_{s, \mu}^{2}-\left[\omega+\left(p n_{b}+\mu\right) \omega_{0}\right]^{2}} .
$$

Let us define $\delta I_{B}(t)$ in terms of the slow functions $\kappa_{b}(t)=a_{B}(t)-i b_{B}(t)$ similarly to Eq. (25),

$$
\delta I_{b}(t)=\frac{\hat{I}_{B}}{2} e^{-i \omega_{g} t} \kappa_{b}[t]+c . c .
$$

The Fourier harmonics

$$
\delta I_{b}(\omega)=\frac{\hat{I}_{B}}{2} \kappa_{-}\left(\omega-\omega_{g}\right)+\frac{\hat{I}_{B}^{*}}{2} \kappa_{+}\left(\omega+\omega_{g}\right)
$$

where

$$
\kappa_{ \pm}(\omega)=a_{B}(\omega) \pm i b_{B}(\omega) .
$$

Neglecting components $\kappa_{ \pm}\left( \pm 2 \omega_{g}\right)$, we get from Eq. (55)

$$
\kappa_{-}(\omega)=\frac{2}{\hat{I}_{B}} \delta I_{B}\left(\omega+\omega_{g}\right), \quad \kappa_{+}(\omega)=\frac{2}{\hat{I}_{B}^{*}} \delta I_{B}\left(\omega-\omega_{g}\right) .
$$

Substituting here Eq. (52), using Eq. (26) and

$$
\frac{\hat{V}_{c}}{\hat{I}_{B}}=\frac{R_{L}}{Y_{L}} e^{-i \phi_{s}}
$$

we get

$$
\begin{aligned}
& \kappa_{-}(\omega)=-\frac{i}{2 \sin \phi_{s}} \sum_{p} \sum_{p^{\prime}} \sigma\left[\omega+\omega_{g}, p\right] e^{-i\left(\frac{\left(p+p^{\prime}\right) n_{b}}{h}\right) \phi_{s}-i \phi_{s}}\left\{U_{-}\left[\omega+\left(p+p^{\prime}\right) n_{b} \omega_{0}\right]\right. \\
& \left.+U_{+}\left[\omega+2 \omega_{g}+\left(p+p^{\prime}\right) n_{b} \omega_{0}\right]\right\}, \\
& \kappa_{+}(\omega)=-\frac{i}{2 \sin \phi_{s}} \sum_{p} \sum_{p^{\prime}} \sigma\left[\omega-\omega_{g}, p\right] e^{-i\left(\frac{\left(p+p^{\prime}\right) n_{b}}{h}\right) \phi_{s}+i \phi_{s}}\left\{U_{-}\left[\omega-2 \omega_{g}+\left(p+p^{\prime}\right) n_{b} \omega_{0}\right]\right. \\
& \left.+U_{+}\left[\omega+\left(p+p^{\prime}\right) n_{b} \omega_{0}\right]\right\} .
\end{aligned}
$$


Because Fourier harmonics of the slow functions $U_{ \pm}(\omega)$ are not zero only at low $\omega$, we can simplify Eq. (59) retaining only the main terms in the sum over $p^{\prime}$ :

$$
\begin{aligned}
& \kappa_{-}(\omega)=-\frac{i}{2 \sin \phi_{s}} \sum_{p} \sigma\left[\omega+\omega_{g}, p\right]\left\{U_{-}(\omega) e^{-i \phi_{s}}+U_{+}(\omega) e^{i \phi_{s}}\right\}, \\
& \kappa_{+}(\omega)=-\frac{i}{2 \sin \phi_{s}} \sum_{p} \sigma\left[\omega-\omega_{g}, p\right]\left\{U_{-}[\omega] e^{-i \phi_{s}}+U_{+}[\omega] e^{i \phi_{s}}\right\} .
\end{aligned}
$$

In the sum $\sum_{p} \sigma\left[\omega \pm \omega_{g}, p\right]$ we can retain terms with small denominators. In this approximation,

$$
\sum_{p} \sigma\left[\omega \pm \omega_{g}, p\right]=\mp \sigma_{ \pm}(\omega)
$$

where

$$
\sigma_{ \pm}(\omega)=\frac{1}{2} \frac{\omega_{s 0}^{2}}{\omega_{s, \mu}^{2}-\omega^{2}}+\frac{1}{2} \sum_{\mu=1-n_{b}}^{n_{b}-1}\left(1 \pm \frac{\mu}{h}\right) \frac{\omega_{s 0}^{2}}{\omega_{s, \mu}^{2}-\left[\omega-\mu \omega_{0}\right]^{2}} .
$$

Let us substitute $\kappa_{ \pm}(\omega)=a_{B}(\omega) \pm i b_{B}(\omega)$ and the definition $U_{p m}(\omega)=u(\omega) \pm i \Phi(\omega)$. Eq. (60) takes the form

$$
\begin{aligned}
& a_{B}(\omega)-i b_{B}(\omega)=\frac{i \sigma_{+}(\omega)}{\sin \phi_{s}}\left\{u(\omega) \cos \left(\phi_{s}\right)-\Phi(\omega) \sin \left(\phi_{s}\right)\right\}, \\
& a_{B}(\omega)+i b_{B}(\omega)=-\frac{i \sigma_{-}}{\sin \phi_{s}}\left\{u(\omega) \cos \left(\phi_{s}\right)-\Phi(\omega) \sin \left(\phi_{s}\right)\right\} .
\end{aligned}
$$

With the accuracy of the terms of the order of $\mu / h, \sigma_{+}(\omega)=\sigma_{-}(\omega) \equiv \sigma(\omega)$. Then, $a_{B}(\omega)=0$ and

$$
\left.b_{B}(\omega)=\sigma(\omega)\left[-u_{(} \omega\right) \cot \left(\phi_{s}\right)+\Phi(\omega)\right] .
$$

The phase-to-phase beam transfer function is $T_{B V}$ defined as $b_{B}(\omega)=T_{B V} \Phi(\omega)$. Therefore, $T_{B V}=\sigma(\omega)$ or

$$
T_{B V}=\frac{1}{2} \frac{\omega_{s 0}^{2}}{\omega_{s, \mu}^{2}-\omega^{2}}+\frac{1}{2} \sum_{\mu=1-n_{b}}^{n_{b}-1} \frac{\omega_{s 0}^{2}}{\omega_{s, \mu}^{2}-\left[\omega-\mu \omega_{0}\right]^{2}} .
$$

The first term here is the Pedersen's beam transfer function. The sum defines contribution of the $\mu \neq 0 \mathrm{CB}$ modes. 


\subsection{Dispersion equation}

The perturbation of the total current is related to $\delta I_{B}(\omega)$ by Eq. (28),

$$
\delta I_{\text {tot }}(\omega)=-R(\omega) \delta I_{B}(\omega)
$$

where $R(\omega)=Z_{H}(\omega) / Z_{c}(\omega)$. Eq. (66) gives after substitution of Eq. (26) and Eq. $(55)$

$$
\hat{I}_{t o t} J_{-}\left(\omega-\omega_{g}\right)+\hat{I}_{t o t}^{*} J_{+}\left(\omega+\omega_{g}\right)=-R(\omega)\left[\hat{I}_{B} \kappa_{-}\left(\omega-\omega_{g}\right)+\hat{I}_{B}^{*} \kappa_{+}\left(\omega+\omega_{g}\right)\right] .
$$

Because $J_{ \pm}(\omega)$ and $\kappa_{ \pm}(\omega)$ are Fourier harmonics of the slow functions, Eq. (67) can be separated in two for $\omega \simeq \pm \omega_{g}$ :

$$
J_{-}(\omega)=-R\left(\omega+\omega_{g}\right) \frac{\hat{I}_{B}}{\hat{I}_{t o t}} \kappa_{-}(\omega), \quad J_{+}(\omega)=-R\left(\omega-\omega_{g}\right)\left(\frac{\hat{I}_{B}}{\hat{I}_{\text {tot }}}\right)^{*} \kappa_{+}(\omega) .
$$

From Eq. (9), $\hat{I}_{\text {tot }}=\hat{V}_{c} / Z_{H}\left(\omega_{g}\right)$. Eq. (8) gives $\hat{I}_{B}=2 I_{d c} e^{i \phi_{s}}$. Therefore, using the definition of $Y_{L}$,

$$
\frac{\hat{I}_{B}}{\hat{I}_{t o t}}=\frac{Y_{L}}{R_{L}} Z_{H}\left(\omega_{g}\right) e^{i \phi_{s}} .
$$

Substitute Eq. (60) for $\kappa_{ \pm}(\omega)$ and use definition of $J_{ \pm}, U_{ \pm}$of Eq. (26). That gives $a(\omega)$ and $(\omega)$ in terms of $u(\omega)$ and $\Phi(\omega)$ :

$$
\begin{aligned}
& a(\omega)=i K_{-}\left[u(\omega) \cot \left(\phi_{s}\right)-\Phi(\omega)\right], \\
& b(\omega)=K_{+}\left[u(\omega) \cot \left(\phi_{s}\right)-\Phi(\omega)\right],
\end{aligned}
$$

where

$$
K_{ \pm}=\frac{Y_{L}}{2 R_{L}}\left[ \pm R\left(\omega+\omega_{g}\right) Z_{H}\left(\omega_{g}\right) \sigma_{+}(\omega) e^{i \phi_{s}}+R\left(\omega-\omega_{g}\right) Z_{H}^{*}\left(\omega_{g}\right) \sigma_{-}(\omega) e^{-i \phi_{s}}\right] .
$$

Combining Eq. (70) and Eq. (66), we obtain the homogeneous system of two linear equations. The nontrivial solution exists if the determinant of the system is equal to zero what gives the dispersion equation for $\omega$,

$$
\left.\left.1+i \frac{Y_{L}}{2 R_{L} \sin \left(\phi_{s}\right)}\left\{R\left(\omega+\omega_{g}\right)\right) \sigma_{+}(\omega) Z_{c}\left[\omega_{g}+\omega\right]-R\left(\omega-\omega_{g}\right)\right) \sigma_{-}(\omega) Z_{c}\left[\omega-\omega_{g}\right]\right\}=0 .
$$

In terms of the Laplace variable $s=-i \omega$, Eq. (72) takes the form 


$$
\left.1+i \frac{Y_{L}}{2 R_{L} \sin \left(\phi_{s}\right)}\left\{R\left(s-i \omega_{g}\right) \sigma_{+}(s) Z_{c}\left[s-i \omega_{g}\right]-R\left(s+i \omega_{g}\right)\right) \sigma_{-}(s) Z_{c}\left[s+i \omega_{g}\right]\right\}=0 .
$$

Here $R(s)=Z_{H}(s) / Z_{c}(s), Z_{H}(s)$ is equal to $Z_{c}(s)$ if $H=0$, and

$$
\begin{aligned}
& \sigma_{ \pm}(s)=\frac{1}{2} \frac{\omega_{s 0}^{2}}{\omega_{s 0}^{2}+s^{2}}+\frac{1}{2} \sum_{\mu=1-n_{b}}^{n_{b}-1}\left(1 \pm \frac{\mu}{h}\right) \frac{\omega_{s 0}^{2}}{\omega_{s, \mu}^{2}+\left(s+i \mu \omega_{0}\right)^{2}} \\
& Z_{H}(s)=R_{L}\left[\frac{\sigma_{r}}{s+i \omega_{c}+\sigma_{r}\left(1+H e^{\left.-\left(s+i \omega_{c}\right) \tau_{d}\right)}\right.}+\frac{\sigma_{r}}{s-i \omega_{c}+\sigma_{r}\left(1+H e^{\left.-\left(s-i \omega_{c}\right) \tau_{d}\right)}\right.}\right],(
\end{aligned}
$$

where $\sigma_{r}=\omega_{c} /\left(2 Q_{L}\right)$.

If only a single coupled-bunch mode $\mu=0$ is taken into account, then $\sigma_{-}(\omega)=\sigma_{+}(\omega)$ what is the same as the Pedersen's beam transfer function $B(s)=\omega_{s 0}^{2} /\left(\omega_{s 0}^{2}+s^{2}\right)$.

\section{Analysis of stability}

The dispersion equation Eq. (72) generalize Pedersen's result including all CB mode. The system is stable if all eigenvalues $\omega$ have negative imaginary parts. In terms of $s=-i \omega$, the growth rate $\Gamma_{\mu}=R e[s]$.

As an example, we analyze stability of the LER of PEP-II B-factory. The main parameters of the system are given in Table 1 . Harmonic number $h=2 n_{b}$. The optimum calculated steady-state parameters are given in Table 2.

\section{Table 1: Parameters of the LER PEP-II.}

\begin{tabular}{|l|l|l|}
\hline \hline$n_{\text {cav }}$ & & 6. \\
$f_{r f}$ & $\mathrm{MHz}$ & 476. \\
$V_{c} /$ cavity & $\mathrm{MeV}$ & 0.85 \\
$R_{0}$ & $\mathrm{MOhm}$ & 3.5 \\
$Q_{0}$ & & $3.010^{4}$ \\
$U_{\text {loss }}$ & $\mathrm{MeVr}$ & 0.77 \\
$I_{d c}$ & $\mathrm{Amp}$ & 2.25 \\
$N_{B}$ & $10^{10}$ & 5.8 \\
$s_{b}$ & $\mathrm{~m}$ & 1.24 \\
$n_{b}$ & & 1658 \\
\hline
\end{tabular}

The growth rate was calculated by solving numerically the dispersion equation Eq. (72), (73) using MATHEMATICA. 
Table 2: The steady-state parameters.

\begin{tabular}{|l|c|c|}
\hline \hline$\beta$ & & 3.909 \\
$Y /(1+\beta)$ & & 3.774 \\
$Q_{L}$ & & 6111. \\
$R_{L}$ & MOhm & 0.712 \\
$\phi_{s}$ & degree & 80.96 \\
$\psi$ & degree & 74.95 \\
$f_{s}$ & $\mathrm{kHz}$ & 4.5 \\
$\left(\omega_{c}-\omega_{g}\right) / \omega_{0}$ & & 1.064 \\
$\tau_{d}$ & $\mathrm{~ns}$ & 450 \\
\hline
\end{tabular}

Figs. (5-7) show results of calculations. Fig. (5) gives results with only $\mu=0$ mode taken into account for several values of the gain $H$. The upper pane gives the growth rate $\Gamma$ with the $\mathrm{FB}$ off $(H=0)$ and for single mode taken into account $\left(\mu_{\max }=0\right)$. The result in this case is exactly the same as given by the Pedersen dispersion equation. The next panes show how the growth rate is modified with the increasing gain. The threshold of instability increases with $H$ and instability is completely suppressed at $H=6$. Fig. (6) shows effect of one additional mode $\mu_{\max }=1$ taken into account for $H=0, H=3$, and $H=6$. The instability starts from the zero current as it is suppose to do for the coupled-bunch case. Fig. (7) gives similar result for four CB modes taken into account $\mu_{\max }=3$. In all cases the delay time of the $\mathrm{FB}$ is $\tau_{d}=450 \mathrm{~ns}$.

\section{Conclusion}

F. Pedersen analysis gives the foundation for the design of the low-level rf feedback system. In his analysis the beam response is described by the beam transfer function which takes into account the single $m=0 \mathrm{CB}$ mode. We generalize this analysis defining in Eqs. (72),(73) the dispersion equation taking into account all CB modes. The growth rate for the PEP-II was calculated by solving dispersion equation numerically using MATHEMATICA with a single $m=0 \mathrm{CB}$ mode $\left(\mu_{\max }=0\right)$ and with several $\mathrm{CB}$ modes. The difference of the results shows effect of the unstable CB modes.

\section{Acknowledgement}

We appreciate discussions with J. Fox and C. Riretta.

Work supported by Department of Energy contract DE-AC03-76SF00515. 


\section{References}

[1] F. Pedersen, Beam loading effects in the CERN PS booster, IEEE in Nucl. Science, Vol. NS-22, No. 3, June 1975

[2] F. Pedersen, RF cavity feedback, PEP-II reports

[3] P. B. Wilson, High energy electron linacs: applications to storage ring RF systems and linacs, SLAC-PUB-2884, Nov. 1991

[4] G. Kraft, private communication.

[5] PhD Thesis.

[6] S. Heifets, A.Chao, Collection of formulas describing storage ring coherent instabilities, SLAC-PUB-10167, September, 2003

[7] A.W. Chao Physics of collective beam instabilities in high energy accelerators, J. Wiley and Sons, inc, 1993 


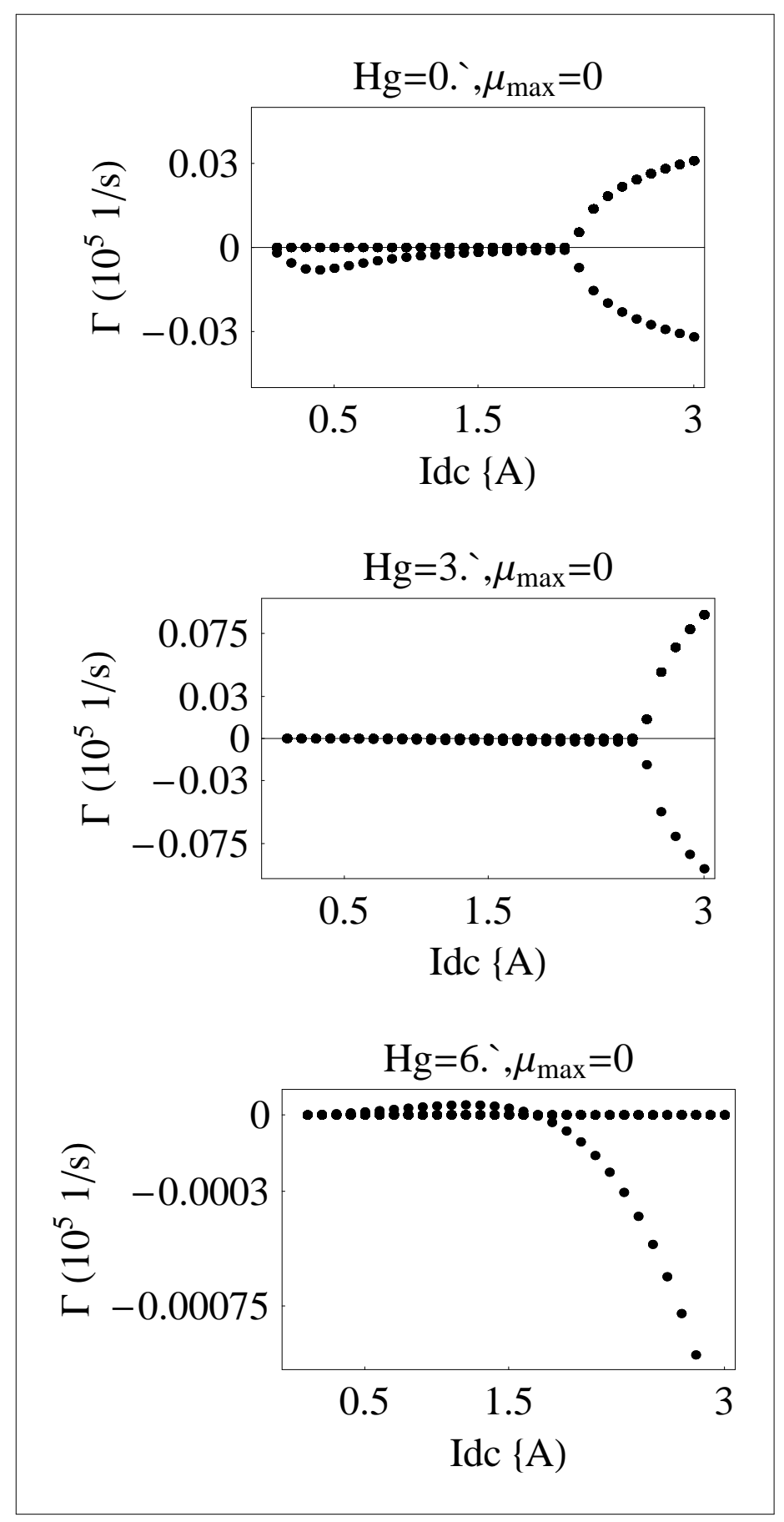

Figure 5: 


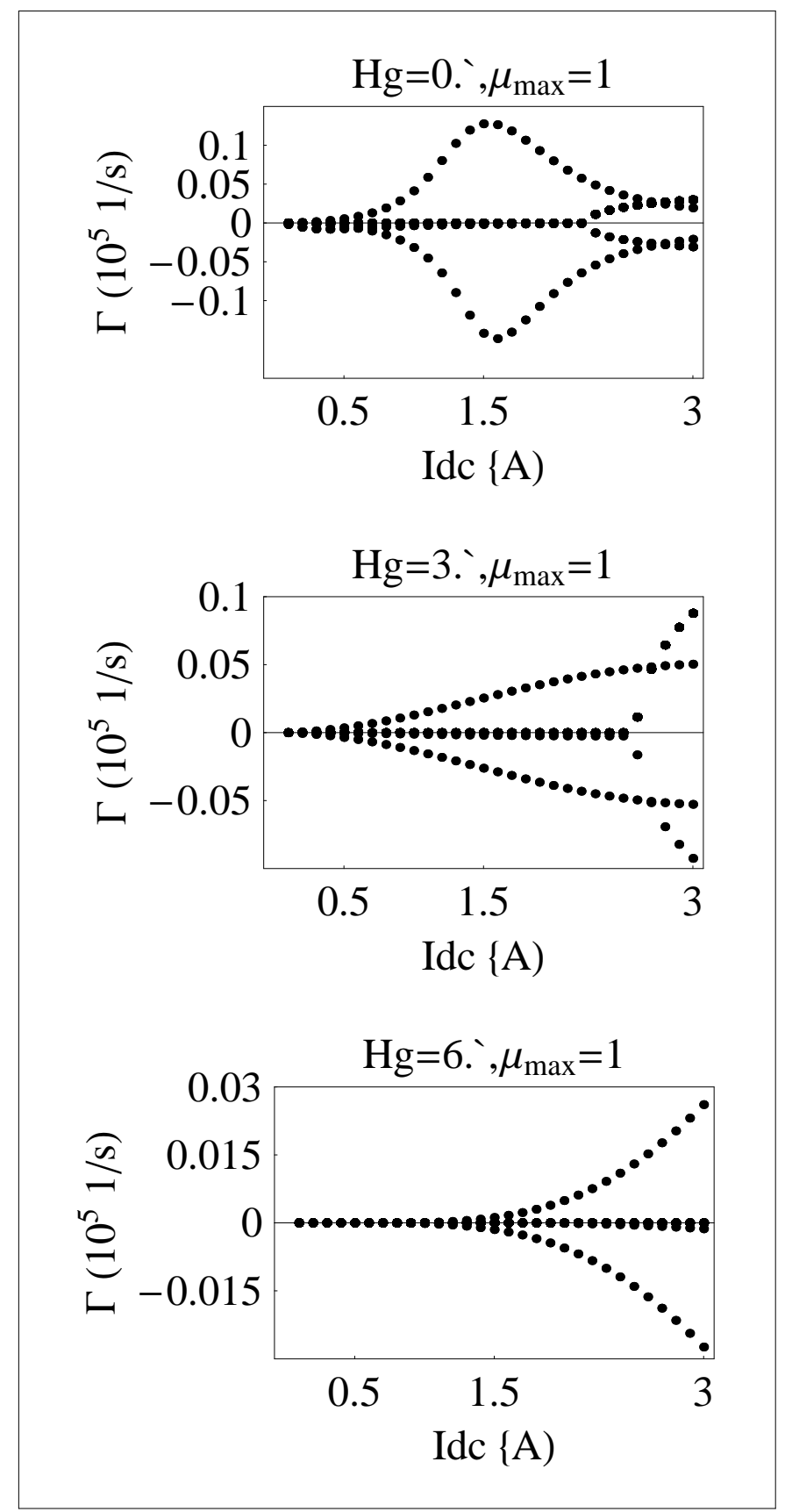

Figure 6: 


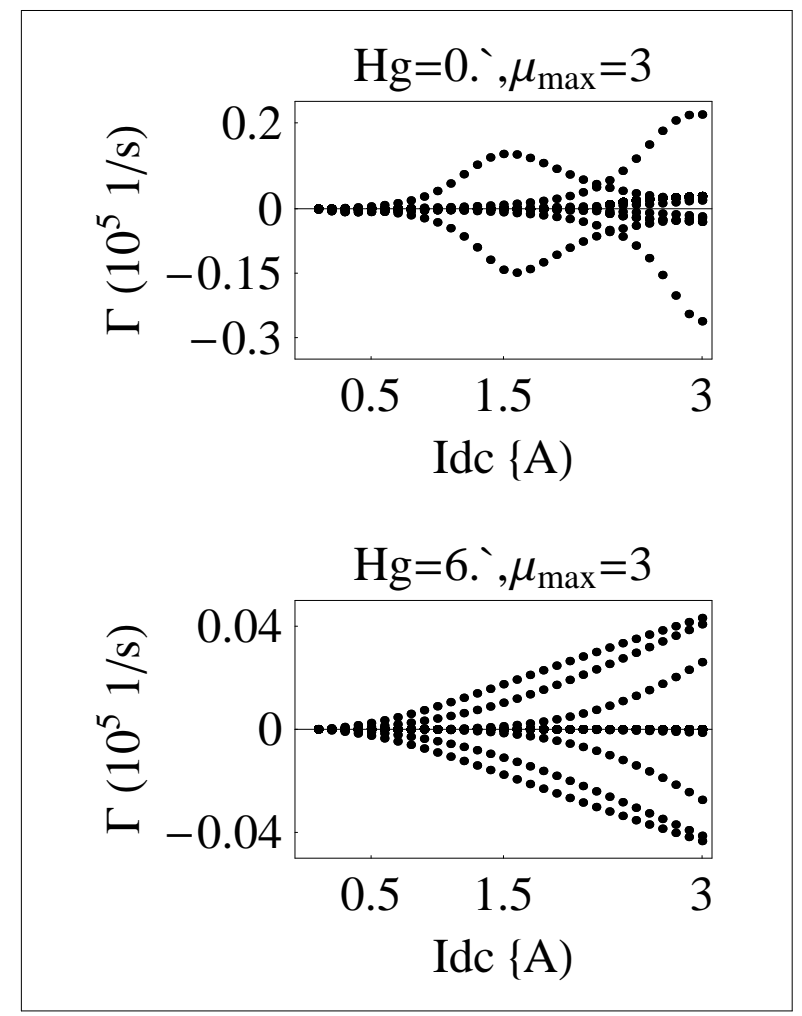

Figure 7: 